\title{
Features of Phase Separation in Polymeric Systems: Cloud-Point Curves (Discussion)
}

\author{
Vitaly J. Klenin, Sergei L. Shmakov* \\ Saratov State University, Institute of Chemistry, Chair of Polymers, 83 Astrakhanskaya st., Saratov 410012, Russian Federation \\ *Corresponding Author: ShmakovSL@info.sgu.ru
}

Copyright $@ 2013$ Horizon Research Publishing All rights reserved.

\begin{abstract}
The paper discusses the complexity and ambiguity of interpretation of plotting binodals (for binary monomolecular polymer + low-molecular-weight liquid systems) or phase separation boundaries (those with a polymolecular polymer) by means of fixing a certain turbidity level when the configurative point moves from the single-phase range towards the phase separation one (the so-called cloud-point curve technique).
\end{abstract}

Keywords Phase Separation, Binodal, Spinodal, Cloud Point, Polymer

\section{Introduction}

State diagrams, or the phase diagrams of multicomponent systems, underlie modern materials science. This fundamental statement fully applies to polymer-containing (or polymeric) systems [1-5].

The usual polymolecularity of polymeric substance, high molecular weights, conformational features of macromolecules determine the high viscosity of polymeric systems and the kinetic retardation of phase delamination [3].

Therefore, to achieve equilibrium liquid-liquid phase separation requires long high-precision thermostating of the system during days, weeks, and months; that is why such measurements have been made for few polymeric systems only. The so-called cloud-point technique is most frequently applied for the phase analysis of polymeric systems, it "consists in determination of the temperature of the initial cloudiness of a solution, which is regarded as the temperature of phase separation $\left(T_{\mathrm{ps}}\right) \ldots$ The temperature of the occurrence of steady cloudiness fixed visually is accepted as the temperature of delamination... Above $80 \%$ of all state diagrams of polymeric systems reported hitherto have been obtained by virtue of this technique", [2].

Wohlfarth [5] formulates the essence of this technique as follows: "The point where first deviations from a basic line are detected is the cloud-point", which is apparently quite similar to the above definition. In the fundamental database [5], in "Chapter 3. Liquid-liquid equilibrium (LLE) data of aqueous polymer solutions; 3.1 Cloud-point and/or coexistence curves of quasibinary solutions", of 68 systems 61 ones were studied by the cloud-point technique, and only 5 systems were analyzed by the coexistence curve technique.

The cloud-point technique, besides some principal difficulties (to be discussed below), has purely technical and procedural uncertainties. In particular, the turbidity level observed by the operator, besides the analytical turbidity of the system, is dependent on the volume and shape of the vessel, the background color behind this vessel, room illumination, not to mention the psychological and ophthalmologic state of the operator himself. Symptomatically, the authors of papers reporting the results of turbidimetry application, as a rule, avoid description of any details of their cloud-point procedure.

On our analysis of a large number of papers involving turbidimetry, an impression is made that the majority of researchers do not realize the ambiguity and complexity of interpretation of the results of this approach in the analysis of polymeric systems. The present paper is aimed at improvement of the situation in the matter.

To start discussion of this problem, let Fig. 1 present experimental data obtained by this technique for a specific system [polystyrene (PS) + di(2-etylhexyl) phthalate (DEHP)] [6], which are accepted as initial data for further studies on this system [6] and have entered a regular database for the phase diagrams of polymeric systems [4].

However, even a fluent sight at these data is enough for judgment about their discrepancy to the most general statements of the phase separation theory of the P+LMWL system, according to which, when the molecular weight of the polymer increases, the binodal and spinodal of the P+LMWL system with an UCST get narrower with an increase in the critical temperature $T_{\text {cr }}$ up to $T_{\text {cr }}=\theta$ and a decrease in the critical concentration $c_{\text {cr }}$ down to zero $[8,9]$.

\section{Theoretical analysis}


For more detailed analysis, we have plotted the binodals and spinodals according to the Flory-Huggins theory (the first approximation) [8,9] for this system. The adequacy of this model is determined by the nonpolarity of the polymer and the weak polarity of the solvent. There are no H-bonds in the system. In view of the molecular weight of a PS monomer link, the molar volume of DEHP, and the molecular weight of PS samples (1-4 in the ascending order) [6] the number of cells in the lattice model was accepted as $z$ $=342,900,1500$, and 18,000, respectively. For more easy-to-grasp comparison with the theory, we have conditionally accepted the experimental points of the lowest-molecular-weight PS sample as the left branch of the binodal, the more so that of all the experimental curves this one is most similar to the binodal qualitatively, which is reflected in a good fit to the experimental points of the calculated binodal with the interaction parameter

$$
\chi_{1}=\frac{62}{T}+0.317
$$

Regression of this binodal was made by the algorithm offered in [10].

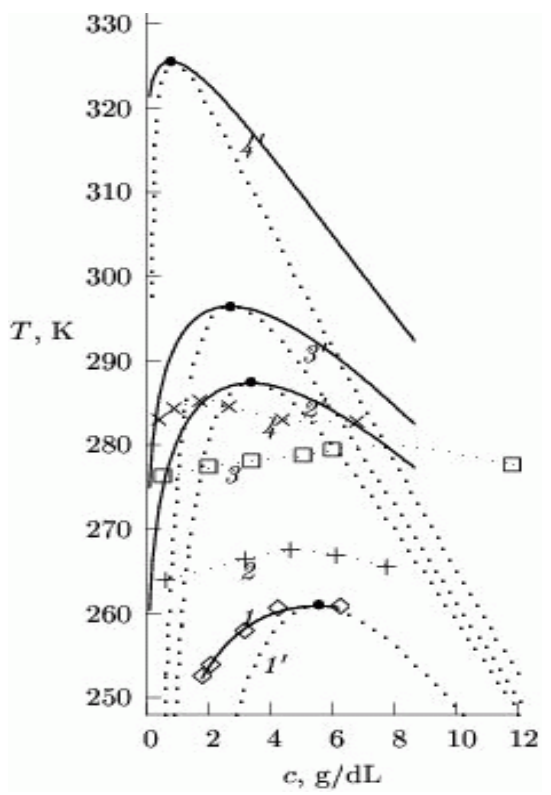

Figure 1. Experimental cloud points in the polystyrene + di(2-ethylhexyl) phthalate system, the data by Vshivkov and Rusinova [6]. The molecular weight of polystyrene samples is: 57 (1); 160 (2); 250 (3); and 3,000 $\mathrm{kDa}(4)$. Calculated binodals (solid curves) and spinodals (dotted curves) for this system with $z=342\left(1^{\prime}\right)$; $960\left(2^{\prime}\right) ; 1,500\left(3^{\prime}\right)$; and 18,000 (4').

Fig. 1 presents the calculated binodals (solid curves) and spinodals (dotted ones) of the PS+DEHP system for the PS samples with $z=342$ (curves $1^{\prime}$ ), 960 (2'), 1,500 (3'), and $18,000\left(4^{\prime}\right)$. As can be expected, the distinction between the experimental points and theoretical binodals is impressing.

With the purpose of further analysis of the situation, the turbidity isotherms $\tau$ of solutions and the diffusion coefficients $D$ of PS macromolecules at $T=258 \mathrm{~K}$ are drawn in Fig. 2, while Fig. 3 shows the temperature dependence $\tau=$
$\tau(T)$ of solutions of the four PS samples for $c=3.2 \mathrm{~g} / \mathrm{dL}$.

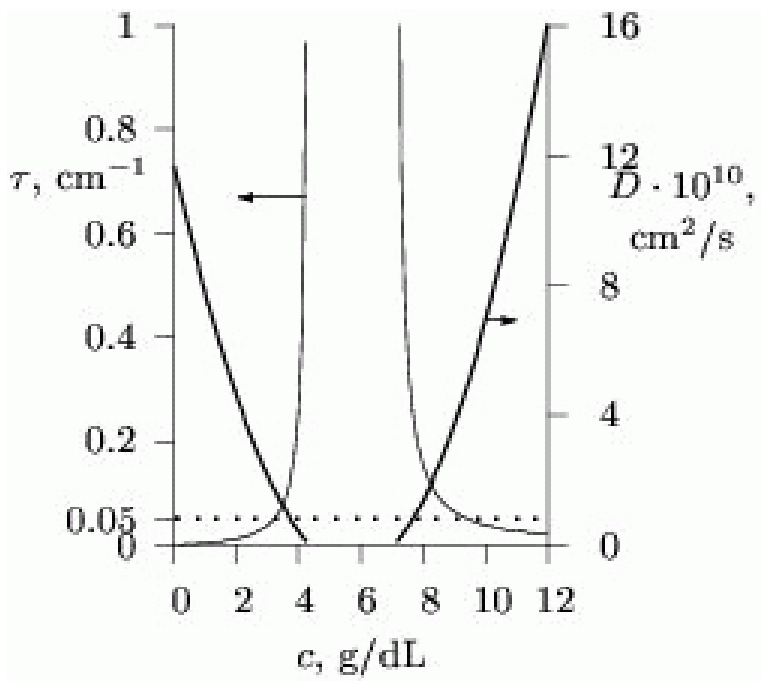

Figure 2. Concentration dependence of the calculated turbidity $\tau, \mathrm{cm}^{-1}$, and the diffusion coefficient $D$ of macromolecules for a PS solution with $M$ $=57 \mathrm{kDa}(z=342)$ at $258 \mathrm{~K}$.

The turbidity of solutions was calculated by the Einstein —Debye-Bueche formula [11]:

$$
\tau=\frac{H R T \bar{V}_{01} c}{\partial\left(\pi \bar{V}_{01}\right) / \partial \mathrm{c}}=-\frac{H R T \bar{V}_{01} c}{\partial \mu_{1} / \partial \mathrm{c}},
$$

where

$$
H=\frac{32 \pi^{2} n^{2}}{3 \lambda_{0}^{4} N_{A}}\left(\frac{\partial n}{\partial c}\right)^{2} .
$$

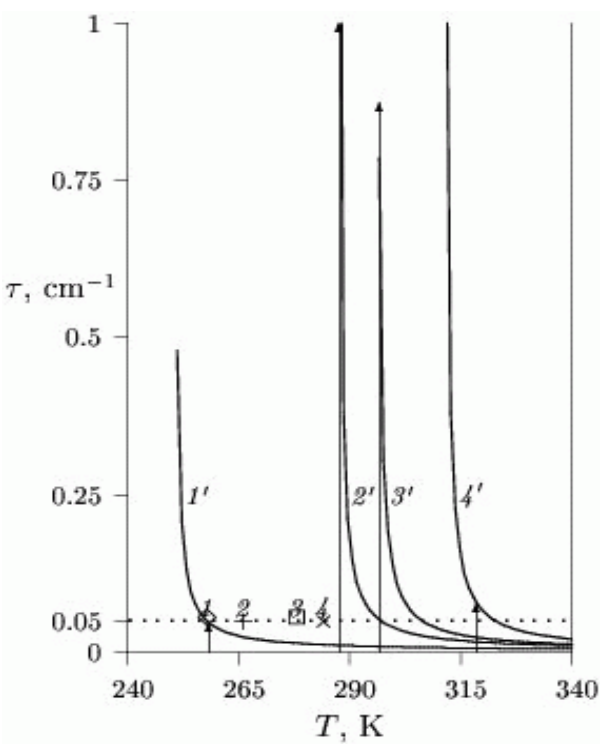

Figure 3. Temperature dependence of the turbidity of the solutions of PS samples $\left(1^{\prime}-4^{\prime}\right)$ with a concentration $c=3.2 \mathrm{~g} / \mathrm{dL}$. Digits $1-4$ designate the cloud-point temperatures for solutions of PS samples $(1-4)$ with $c=3.2 \mathrm{~g} / \mathrm{dL}$ by the data from [6] (Fig. 1). The vertical straight lines with arrows show the calculated binodal temperatures (see Fig. 1). 
The diffusion coefficient $\mathrm{D}$ was calculated by the formula

$$
D=\frac{x_{1} M}{N_{A} f_{2}}\left(\frac{\partial \pi}{\partial c}\right)=-\frac{x_{1} M}{N_{A} f_{2} \bar{V}_{01}}\left(\frac{\partial \mu_{1}}{\partial c}\right)
$$

(see (3.3-12) in [3]).

Therefore, any characteristic features of $\tau$ and $D$ are determined by the behavior of the derivative $\partial \mu 1 / \partial \mathrm{c}$ since

$$
\tau \sim-\left(\frac{\partial \mu_{1}}{\partial c}\right)^{-1}, \quad D \sim-\frac{\partial \mu_{1}}{\partial c},
$$

which has decisive importance in experiments with solutions, in general, and with polymer solutions, in particular, in which the configurative point moves along an isotherm (Fig. 2) or along the temperature axis at $\mathrm{c}=$ const (Fig. 3). It follows from (3) that $\tau \rightarrow \infty$ and $\mathrm{D} \rightarrow 0$ on the spinodal.

\section{Experiment-Theory Comparison}

It follows from Fig. 2 that the binodal concentration $c_{\mathrm{I}}$ at $258 \mathrm{~K}$ for the first PS sample is not a special point on the $\tau=$ $\tau(c)$ dependence as well as the binodal temperature at $c_{\mathrm{I}}=3.2$ $\mathrm{g} / \mathrm{dL}$ on the $\tau=\tau(T)$ dependence for the same sample (Fig. $3)$.

If curve 1 in Fig. 1 is accepted as the binodal, this means that in this specific performance of the technique the turbidity value $\tau=0.05 \mathrm{~cm}^{-1}$ was chosen for the level of "steady cloudiness". Working with the device, the operator sets, say, this level $\tau=0.05 \mathrm{~cm}^{-1}$ and fixes it for the whole series of experiments or even for the whole procedure. Having fixed this turbidity level for other PS samples, Vshivkov and Rusinova [6] have obtained $T \approx 266,278$, and $284 \mathrm{~K}$ (see Figs. 1 and 3) as the binodal temperatures for $c=$ $3.2 \mathrm{~g} / \mathrm{dL}$, but should, in idea, have fixed $T=297,306$, and $323 \mathrm{~K}$ (see Fig. 3). Fig. 3 implies the incorrectness of fixing binodal temperatures by achieving any certain turbidity level, as the binodal temperature has always (!) different turbidity levels depending on the degree of remoteness of this solution concentration from the critical concentration of the system. This is well seen from Fig. 3. If for sample 4 the turbidity level is somewhere comparable with the conditionally (!) accepted threshold of $0.05 \mathrm{~cm}^{-1}$, the binodal temperature for samples 2 and 3 correspond to an indefinitely high turbidity, as at $c=3.2 \mathrm{~g} / \mathrm{dL}$ the binodal is very close to the spinodal (see Fig. 1). Fixing the temperature of any certain turbidity level in solutions with a concentration close to the critical one (unknown, as a rule), the operator will inevitably overestimate the binodal temperature. Vshivkov and Rusinova [6], however, obtained reverse results, because there were a plenty of causes for such artifacts in the procedure of "steady cloudiness" as well.

Hitherto, the thermodynamic aspects of the problem were discussed, but, as "steady cloudiness" is fixed when the configurative point moves with a finite rate, there appear problems of kinetic character. When crossing the binodal, the configurative point penetrates into the range of metastable states of the solutions, in which the system will inevitably stay during the induction period of an unknown duration. It is only certainly known that near the binodal (already from the side of the metastable region) the induction period is very long - theoretically, infinitely long. In view of this, it is basically impossible to fix a point on the binodal when the configurative point moves from the stable range of a solution with a finite rate.

In this connection, it seems expedient to cite [12], where the authors show a great irreproducibility of cloud points when polyacrylamide (PAA) aqueous solutions are titrated with acetone and the invalidity of the solubility equation

$$
\gamma=-\frac{A}{\sqrt{M}} \ln c_{1}+\frac{B}{\sqrt{M}}+\gamma_{\vartheta},
$$

where $\gamma$ is the volume fraction of the non-solvent, $\gamma_{\theta}$ is the same at the $\theta$ composition, $c_{\mathrm{I}}$ the polymer concentration at the left-hand binodal branch ( $T=$ const), $M$ is the molecular weight of the polymer, $A$ and $B$ are constants.

In the PAA+acetone system, higher cloud points $\gamma$ for the high-molecular PAA samples than for the low-molecular ones were found [12]. Solutions of high-molecular fractions could not often be titrated, i.e. they remained transparent at a sufficiently high content of acetone $(\gamma \rightarrow 1)$. However, in hours or days, the systems spontaneously grew turbid. These facts attest to the system being "inclined" to oversaturation owing to a long induction period of the formation of new-phase particles (high-molecular PAA fractions).

Klenina and Lebedeva [12] finally succeeded in finding a technique (even two ones were proposed) of obtaining the equilibrium values of cloud points in this system. The first technique envisages adding acetone in excess amounts, waiting for phase separation, and performing back titration, i.e. turbidity was measured on adding water to fix the point where it vanishes (the system gets transparent). The value of $\gamma$ at which $\tau \rightarrow 0$ was accepted as the phase separation region boundary. This technique gave well-reproducible results. Moreover, such well-reproducible $\gamma$ 's were obtained with direct turbidimetric titration and well as with the back one provided that a small amount of $\mathrm{NaCl}\left(0.5<c_{\mathrm{s}}<20 \mathrm{mM}\right)$ was added.

Special experiments established no influence of small amounts of $\mathrm{NaCl}$ on the location of the boundary of the phase separation region. Perhaps, the ions of $\mathrm{NaCl}$ played the role of nuclei of a new phase and drastically shortened the induction period.

On establishing the phase separation region boundary within $10^{-6}<v_{2 \mathrm{I}}<10^{-3}$, Klenina and Lebedeva [12] determined the $\theta$ composition of the water+acetone mixture by Elias' method (see (4)): $\gamma_{\theta}=0.32$, confirmed by light scattering $\left(A_{2}=0\right)$.

In the PAA+water+acetone system, a coil-to-globule transition was observed $[13,14]$.

Baranovskaya et al. [14] established a sharp (300-fold) decrease in the macromolecular volume (with the molecular 
weight remaining constant) in the branched PAA $\left(M_{\mathrm{w}}=30\right.$ $\mathrm{MDa}$ +water+acetone system at the volume fraction of acetone $\gamma=0.35$ by means of classical light scattering.

These authors, as well as Nishio et al. [13], reported no boundary of the liquid-liquid phase separation region and no $\theta$ composition in the $\mathrm{PAA}+$ water+acetone system. This might possibly be due to the system being "inclined" to oversaturation at high molecular weights, which hinders obtaining the phase separation range border [12].

Comparison of the value $\gamma_{\theta}=0.32$ and the location of the phase separation range border on a quasibinary section [12], on the one hand, and the experimental conditions in [13] and [14], on the other hand, suggests that the coil-globule transition is observed not only when $\gamma>\gamma_{\theta}$, but also when $\gamma$ exceeds the value $\gamma$ of the boundary curve, i.e. in the liquid-liquid phase separation region. A similar situation was observed for the PAA+water+methanol system as well [12, 15].

Recall that the thermodynamically equilibrium coil-globule transition must occur between the $\theta$ temperature (the $\theta$ composition) and the phase separation range border. Hence, it was observed [13, 14] in the metastable region of the liquid-liquid phase transition and with an essential oversaturation with respect to the coil—globule transition. Technically, such a tendency of the system to oversaturation enhances the possibility to observe the coil-globule transition with respect to the concentration scale of polymer and precipitant. This may serve as an explanation why the most significant effects of the coil-globule transition have been found in systems with oversaturation (in a "foreign field").

The coil-globule transition proceeding under conditions of oversaturation counts in favor of its being close to the first-order transition.

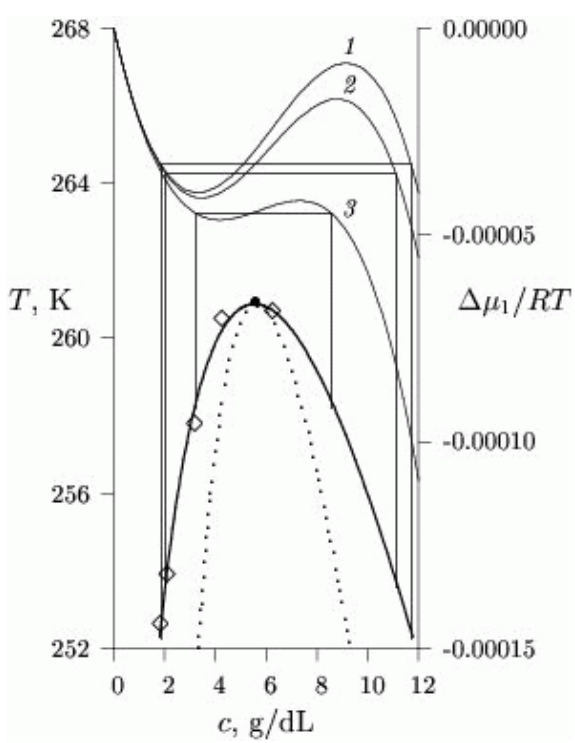

Figure 4. Chemical potential of mixing of the solvent $\Delta \mu_{1} / R T$ for the PS (sample 1) + DEHP system at $T=252.6$ (1), 253.9 (2), and $257.8 \mathrm{~K}$ (3). Binodal (solid curve) and spinodal (dotted curve) for this system.

In the metastable range, the progressively growing (see
Fig. 3) turbidity of the system caused by concentration fluctuations may at any moment (unknown!) be supplemented by the turbidity due to the formation of particles of a new phase. Therefore, it is principally impossible to understand what exactly the operator fixes by measuring the temperature of any previously chosen turbidity level. And the list of difficulties is still incomplete! When the configurative point is moving along either an isotherm or the temperature axis at $c=$ const towards the phase separation range, $\partial \mu_{1} / \partial c$ decreases and vanishes near the spinodal (Fig. 4). In this area of the state diagram, $D$ tends to zero, from Eqs. (2) and (3).

Concentration fluctuations play the major role in the problem under discussion. The dynamics of these fluctuations obeys Onsager's principle [16], according to which, spontaneously arisen concentration fluctuations $\delta \mathrm{c}$ disappear under macrophysic laws, i.e. by Fick's laws:

$$
\vec{L}=-D\left(\frac{\partial \delta c}{\partial x}\right)
$$

and

$$
\frac{\partial \delta c}{\partial t}=D \frac{\partial^{2} \delta c}{\partial x^{2}}
$$

This implies that near the spinodal (in particular, near the critical point) the spontaneously arisen concentration fluctuations almost do not disappear because of $D \rightarrow 0$, i.e. the system is "frozen" or, speaking in the modern computer jargon, "hangs", with no reaction to changes in external conditions. That is why Semenchenko [17] stated the principal unattainability of the critical point of a system.

The following experimental facts give evidence of the effect of "frozen" concentration fluctuations. At spectroturbidimetric titration of polymer solutions, particles of the precipitated polymer of an almost equal average size are formed for different polymer + solvent + precipitant systems, corresponding to $n=2$ in the $\tau=A \lambda^{-n}$ equation, which follows from the theory of light scattering from solutions in the critical opalescence range [3]. When the configurative point crosses the critical opalescence range of solutions, owing to the effect of critical retardation, the arisen concentration fluctuations do not disappear and actually pass into particles of the precipitated polymer. A similar effect of critical retardation could also explain the "delay" of the occurrence of new-phase particles, this occurrence being false fixed as the binodal by the "steady cloudiness" technique (see Fig. 1).

There exists another cause of the untypical behavior of the PS samples (Fig. 1), namely, their probable polymolecularity. According to the Flory-Huggins theory [8, 18], polymer samples obtained by consecutive precipitation or dissolution contain all fractions of the initial polymer but in different ratios; therefore, such samples could be actually even more polymolecular than the initial polymer. In such a case, the state diagram in the $T$ vs. $c$ coordinates comprises 
characteristic curves of a quasi-binary section of the polynary $\mathrm{P}+\mathrm{LMWL}$ system: the spinodal and the phase separation range border (PSRB), whose contours strongly depend on the distribution function of the polymer. Certainly, all the aforesaid complications remain in this case as well (and are even amplified).

Therefore, it is principally impossible to fix crossing the binodal (or PSRB) by fixing a common preset (how else it could be done?) turbidity level when the configurative point is moving along the temperature axis at $c=$ const from the single-phase range to the phase separation range in a certain interval of polymer concentrations by virtue of different (and unknown) degrees of remoteness of the solution of the given concentration from the critical concentration of the system and the critical retardation effect.

To raise the sensitivity of locating the cloud point and to improve the reproducibility of results, thin layers of the working cuvette and, correspondingly, low volumes of the system are used (see, e.g. [19]). But in this case, the cuvette surface begins playing a big role, and the experimental results should be treated in terms of the thermodynamics of thin-layer systems [20].

All these difficulties of locating binodal points apply to diffusion coefficients as well (see. Fig. 2), though this technique is used very rarely.

Unlike binodals and PSRB, the spinodals of the P+LMWL systems are determined with less difficulty [21]. The temperature dependence of the inverse intensity of scattered light or that of the inverse turbidity at a given concentration in a wide temperature range looks almost as a straight line (Fig. 5), which provides more or less reliable estimation of the spinodal temperature by the $\tau^{-1} \rightarrow 0$ extrapolation (of course, within the limits of the induction period, if the trajectory of the configurative point traverses the metastable range).

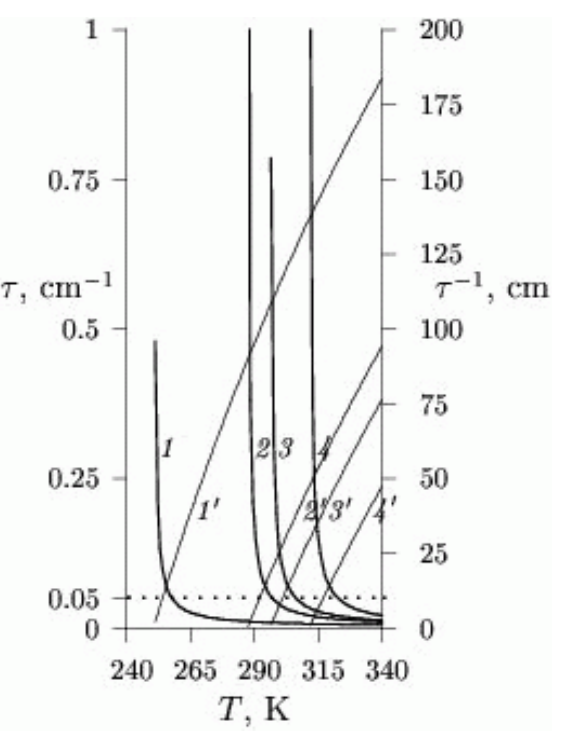

Figure 5. Temperature dependence of turbidity $\tau(1-4)$ and that of the inverse turbidity $\tau^{-1}\left(1^{\prime}-4^{\prime}\right)$ for solutions of PS samples $1-4$.

We note in passing that a spinodal plotted in this way gives a good reference point for estimation of the location of the binodal or PSRB, because these curves are located above the spinodal and have a common (critical) point with it.

For plotting the liquidus curve at liquid — crystal phase separation by the polythermal "steady cloudiness" technique, the situation becomes somewhat simpler because of no precritical phenomena in the liquid - crystal transition (provided that any liquid - liquid phase separation range is remote), however, the complexities of the metastable state remain and have specific features, as not just large enough concentration fluctuations should appear in the system but the occurrence of post-critical nuclei with a structure characteristic of the morphological form of the given crystal, therefore, the induction period of new-phase particle formation can be rather long.

\section{An Alternative Approach}

In view of these complexities of plotting the binodal and PSRB from the single-phase range of the system, the drawing of these curves from the metastable range by the turbidity spectrum technique [3] appears more expedient. In this variant (measuring turbidity at three or four wavelengths on a common-type colorimeter with a thermostated housing), the configurative point is sharply driven inside the metastable range, where new-phase particles are formed. The average size of these new-phase particles, their numeric and weight-volume concentration, i.e. the degree of phase transformation are determined by the turbidity spectrum method. The phase separation rate is measured at several temperatures $V=V(T), c=$ const. The $V(T) \rightarrow 0$ extrapolation gives the temperature of PSRB at the given concentration. The set of thus obtained temperatures in some concentration range of the polymer results in a PSRB, whose shape allows making essentially important conclusions. At liquid-crystal phase separation, the $V(T)$ dependence has extremal character. Then, the $V(T) \rightarrow 0$ extrapolation of the right-hand branch will determine the liquidus temperature $T_{1}$ at the given concentration of the polymer, and that of $V(T) \rightarrow 0$ of the left-hand branch will provide the vitrification temperature $T_{\mathrm{v}}$. The concentration dependence $V(c)$ at $T=$ const, as a rule, has extremal character as well. In this case, the $V(T) \rightarrow 0$ extrapolation of the left-hand branch of $V(c)$ results in the liquidus concentration $c_{1}$ at a given $T$, and that of the right-hand branch leads to the vitrification concentration $c_{\mathrm{v}}$. The character of changes in the average particle size during their formation has criterial importance for the type of phase separation: liquid-liquid or liquid - crystal, and gives important data on the phase separation mechanism [3, 4].

In a simpler version, it is possible to measure the rate of increasing turbidity. The advantage of such an approach consists in that, in this case, the two components of the overall turbidity (those due to concentration fluctuations and new-phase particles) are separated automatically. The turbidity caused by concentration fluctuations, irrespective 
of its scale, is established almost instantly and does not change with time.

\section{Conclusion}

The paper discusses features of the cloud- point technique for plotting the phase diagrams of the polymer (P) +low-molecular-weight liquid (LMWL) systems. The technique consists in fixing a certain, preset or anticipated turbidity level of the system when its configurative point is moving from the single-phase range (solution) towards the two-phase one. The temperature at which the said turbidity level is reached is accepted as the binodal temperature $T_{\mathrm{bi}}$.

It is shown that when temperature decreases in the P+LMWL system with an UCST the solution turbidity, owing to concentration fluctuations, gradually increases up to a maximum at the spinodal temperature $T_{\mathrm{sp}}$. The absence of any feature on the $\tau=\tau(T)$ curve at the binodal temperature is the most important point in this context! From general (theoretical) considerations it is only clear that, as the polymer concentration $c$ gets more and more distant from the concentration $c_{\mathrm{cr}}$, the temperature range between $T_{\mathrm{bi}}$ and $T_{\mathrm{sp}}$ enhances, which means reduction of the turbidity level at $T_{\mathrm{bi}}$ as $c$ moves off from $c_{\mathrm{cr}}$, i.e. the turbidity level at $T_{\mathrm{bi}}$ a priori depends on $c$. Therefore, fixing of the same turbidity level in the phase analysis of the $\mathrm{P}+\mathrm{LMWL}$ system at different $c$ or even in different systems (the same instrument!) inevitably leads to artifacts.

Owing to the high molecular weight of the polymeric component, the turbidity level of such solutions exceeds the turbidity of solutions of low-molecular-weight compounds by few orders of magnitude, so a preset turbidity level may thus be observed in the field of critical opalescence of solution before the phase separation range.

In the situation under discussion the turbidity of the system consists of two components, namely: the turbidity of molecular scattering $\tau_{\mathrm{M}}$ and the turbidity of colloidal scattering on new-phase particles $\tau_{\mathrm{c}}$ which arise not just on crossing the binodal but after the induction period of the metastable state $t_{\text {in }}$ which is very difficult to estimate even for a known system since it depends on many parameters beyond account and analysis (the existence or absence of pollution or impurity particles, the volume and shape of the vessel, possible shakings of the vessel, the influence of the vessel's walls, etc.). From general (theoretical) considerations, only one is known: near the binodal from the two-phase range $t_{\text {in }} \rightarrow \infty$, and near the spinodal $t_{\text {in }} \rightarrow 0$. Hence, the cause of a sharp increase in turbidity at some instant cannot be ascertained in principle: whether this is the increase in the turbidity of molecular scattering at the configurative point approaching the spinodal in the conditions of a metastable state (high $t_{\text {in }}$ ), or this implies the formation of particles in the metastable area after $t_{\text {in }}$ somewhere between $T_{\mathrm{bi}}$ and $T_{\mathrm{sp}}$-but unknown where exactly!

At last, in the critical region (and near the spinodal) the thermodynamic effect of critical retardation (another feature of the critical state along with better known critical opalescence) manifests itself, which is shown in delayed disappearance of concentration fluctuations, which also complicates interpretation of the turbidity behavior when the configurative point moves with a finite rate through the critical range of the system.

The advantages of the turbidity spectrum method in the phase analysis of the P+LMWL systems consists in distinct resolution of two components, namely, molecular and colloidal light scattering.

\section{REFERENCES}

[1] R. Koningsveld, W.H. Stockmayer, E. Nies. Polymer Phase Diagrams: A Textbook. Oxford Univ. Press, New York, 2001 .

[2] S.A. Vshivkov. Research Techniques for Phase Equilibrium in Polymer Solutions, Ural Univ. Press, Sverdlovsk, 1991 (in Russ).

[3] V.J. Klenin. Thermodynamics of Systems Containing Flexible-Chain Polymers, Elsevier, Amsterdam etc., 1999.

[4] A.E. Chalykh, V.K. Gerasimov, Yu.M. Mikhailov. Phase State Diagrams of Polymeric Systems, Janus, Moscow, 1998 (in Russ).

[5] C. Wohlfarth. Handbook of Thermodynamic Data of Aqueous Polymer Solutions, CRC Press LLC, BocaRaton, London, New York, Washington, 2004.

[6] S.A.Vshivkov, E.V. Rusinova. Phase equilibrium of polystyrene solutions in a mechanical field, Vysokomol. soed. A, Vol. 36, No 1, 98-103, 1994 (in Russ).

[7] S.A. Vshivkov, E.V. Rusinova. Phase Transitions in Polymeric Systems Caused by a Mechanical Field, Ural Univ. Press, Yekaterinburg, 2001 (in Russ).

[8] P.J. Flory. Principles of Polymer Chemistry, Ithaca, New York, 1953.

[9] H. Tompa. Polymer Solutions, New York, London, 1956.

[10] S.L. Shmakov. Phase Equilibrium in Solutions of Star-Shaped Macromolecules: an Improved Okada-Numasawa Model, Polymer, Vol. 43, No 4, 1491-1495, 2002.

[11] P. Debye, A.M. Bueche. Light scattering by concentrated polymer solutions, J. Chem. Phys., Vol. 18, No 11, 1423-1425, 1950.

[12] O.V. Klenina, L.G. Lebedeva. Boundary curves of phase separation in the polyacrylamide- water-precipitant system, Vysokomol. soed. B, Vol. 29, No 12, 896-900, 1987 (in Russ).

[13] I. Nishio, Shao-Tang Sun, G. Swislow, T. Tanaka. First observation of the coil-globule transition in a single polymer chain, Nature, Vol. 281, No 5728, 208-209, 1979.

[14] I.A. Baranovskaya, S.I. Klenin, V.A. Molotkov. Observation 
of the coil-globule transition in the polyacrylamide-water-acetone system by light scattering, Vysokomol. soed. B, Vol. 24, No 8, 607-609, 1982 (in Russ).

[15] S.I. Klenin, E.N. Bykova, I.A. Baranovskaya, V.A. Molotkov, V.I. Kurlyankina. A study of the coil-globule transition for high-molecular-weight polyacrylamide by hydrodynamic techniques, Khimiya i fizika vysokomolekulyarnykh soedineniy, 58-59, 1989 (in Russ).

[16] L. Onsager. Reciprocal relations in irreversible processes. I, Phys. Rev., Vol. 37, No 4, 405-426, 1931.

[17] V.K. Semenchenko. On the relation between the thermodynamic stability of a system and the kinetics of the processes proceeding therein, Russ. J. Phys. Chem., Vol. 34, No 6, 1384-1385, 1960 (in Russ).
[18] P.J. Flory. Thermodynamics of heterogeneous polymers and their solutions, J. Chem. Phys., Vol. 12, No 11, 425-438, 1944.

[19] Y.C. Bae, S.M. Lambert, D.S. Soane, J.M. Prausnitz. Cloud-point curves of polymer solutions from thermooptical measurements, Macromolecules, Vol. 24, No 15, 4403-4407, 1991.

[20] A.I. Dolinnyi, B.A. Ogaryov. Surface influence on phase separation in binary systems, Uspekhi khimii, Vol. 57, No 11, 1769-1795, 1988 (in Russ).

[21] Th.G. Scholte. Thermodynamic parameters of polymer-solvent systems from light-scattering measurements below the theta temperature, J. Polymer Sci. A-2., Vol. 9, No 9, 1553-1577 